\title{
NOTAS SOBRE INVESTIGACION FORESTAL: UNA PERSPECTIVA SELVÍCOLA Y DE GESTIÓN
}

\author{
Gregorio Montero González. Presidente de la S.E.C.F. (2005-2013) \\ Felipe Bravo Oviedo. Presidente de la S.E.C.F. (2013-2017) \\ Francisco Javier Silva-Pando. Editor de Cuadernos de la S.E.C.F. (2002-2013)
}

\section{ASPECTOS GENERALES}

La importancia que las diferentes sociedades atribuyen a los servicios que generan los bosques (tanto si son de producción, regulación, culturales o de soporte) varían en función del nivel de desarrollo socio-económico en que se encuentren, del peligro que cada sociedad percibe sobre la integridad y distribución de sus bosques, o la necesidad de asegurar la presencia de estos, en condiciones óptimas, para que garanticen la funcionalidad de los ecosistemas, la conservación de la biodiversidad, la fijación de la población al medio rural, etc.

En muchos casos, especialmente en las zonas con una intensa antropización de los bosques, la gestión forestal sostenible y multifuncional puede crear y conservar estructuras de hábitats más diversos e interesantes para el fomento de la diversidad que las conseguidas por la evolución natural de esos mismos ecosistemas en ausencia de gestión forestal.

En el caso español, el bosque es un componente clave de nuestro paisaje ya que muchas zonas rurales serían inhabitables sin bosques debido a los problemas asociados a corrimientos de tierras, desprendimientos de rocas, torrentes y aludes, etc. En la actualidad, la existencia y cuidado de las masas forestales no obedecen a simples razones de producción, por importantes que estas sean, sino a la necesidad que tienen los países de contar con abundantes y bien distribuidos bosques, ya que éstos representan un papel esencial en el equilibrio biológico y social del territorio (MONTERO et al., 1993).

Los bosques realizan simultáneamente en el tiempo y en el espacio varias funciones sociales, económicas y ambientales vinculadas entre sí. Para garantizar esa multifuncionalidad es necesario aplicar un método de gestión basado en una selvicultu- ra adecuada, lo cual obliga a que la gestión forestal sea cada vez más compleja y menos eficiente en términos de rentabilidad comercial. En algunos casos, se ha confundido multifuncionalidad con la obtención prioritaria de un solo servicio ecosistémico (producción de piña, setas, hongos,..., regulación hidrológica, conservación de especies,...) marginando la obtención de madera. Sin embargo, la corta de árboles no solo permite obtener madera, sino que es la herramienta clave de la selvicultura para modificar las condiciones de los bosques de forma que se puedan obtener otros servicios ecosistémicos. Por tanto, en muchas ocasiones, la corta de árboles se debe incluir como una herramienta clave no solo cuando la obtención de madera sea uno de los objetivos prioritarios dentro del modelo de gestión que se quiere desarrollar.

Los elevados costos de la gestión han propiciado que la demanda de productos forestales y la capacidad de manufacturarlos a bajo coste se hayan trasladado a los países del Este, mientras que la capacidad de producir materias primas a bajo coste se ha trasladado a África y América del Sur (Martínez de ARANO, 2011). Sin embargo, no cabe duda de que el sector forestal en España puede realizar una gran contribución a la economía verde, poniendo en el mercado grandes cantidades de materias primas renovables con gran capacidad de reutilización y contribuyendo a la creación de empleo rural

La investigación en gestión forestal, como medio para la mejora de métodos de aprovechamiento y regeneración, técnicas de producción, modelos de crecimiento, evaluación económica, ambiental y social de los sistemas de gestión sostenible y multifuncionales, no aparece en los grandes programas de investigación que financia el 
sector público, por más que la sociedad y los técnicos forestales exijan, cada vez con más fuerza, la multifuncionalidad de los sistemas selvícolas a aplicar en nuestros montes.

Todos los bosques generan servicios ecosistémicos para la sociedad, pero se entiende por "bosque productor" aquel cuyo objetivo principal es la producción de bienes y servicios con precio de mercado. Con esta definición, en Europa se considera que el $56 \%$ de la superficie de bosque es productora y el resto se distribuye del siguiente modo: un $11 \%$ de bosque protector; un $10 \%$ de bosque para la conservación de la biodiversidad; un $2 \%$ para uso social; un $11 \%$ de uso múltiple-productor, protector y conservador simultáneamente; y el $10 \%$ restante de uso no clasificado (EUROPEAN Commission, 2010-12 y 15).

No es del todo cierto que las sociedades industrializadas hayan prescindido del uso y aprovechamiento de sus bosques con fines económicos, como a veces se afirma de forma general. Todo indica que las sociedades desarrolladas e industrializadas exigen la existencia de bosques multifuncionales y que estos sean gestionados como tales de forma adecuada. La sociedad actual acepta el aprovechamiento económico y sostenido de los bosques, pero exige que los métodos y técnicas de gestión sean sostenibles, multifuncionales y que garanticen, sin ningún género de dudas, la salud y la persistencia del bosque para que pueda seguir cumpliendo el resto de sus funciones ecológicas, paisajísticas y culturales. Tanto en España como en el conjunto de Europa, la gestión forestal se realiza a escala de rodal, o de pequeña propiedad familiar, es decir a escala micro-topográfica y, como ya se ha señalado anteriormente, la gestión debe ser además compleja, lo cual, en ocasiones, dificulta su viabilidad económica (MARTínEZ DE ARANO, 2011). Es decir, las normas básicas dadas por los tratados de selvicultura general y aplicada deben ser matizadas, y muchas veces modificadas en su ejecución final, si se quiere garantizar el equilibrio micro-territorial y las producciones económicas que lo hacen posible. Por otro lado, el cambio de condiciones ambientales que están experimentando los ecosistemas hace que la modificación de las normas básicas antes citadas deba hacerse a través de métodos de gestión adaptativa (BRAVO, 2014). Los márgenes económicos son muy pequeños, de tal forma que ganancias de un 5-10\%, obtenida a través de mejorar la cantidad y calidad de los productos y/o los costes de explotación pueden ser determinantes en el mantenimiento de la actividad.

La mejora de los conocimientos exigidos por la gestión sostenible y multifuncional, que ha de ser aplicada a escala "micro" para que pueda ser eficiente, está fuera de los grandes programas de investigación, y eso a pesar de que afecta a una gran proporción de la superficie de los bosques tanto en España como en la Unión Europea, como antes se ha indicado. En algunos países europeos, con mayor importancia y tradición forestal, se mantiene, en cierta medida, este tipo de investigación en Universidades y Centros de investigación específicos, como parte de su compromiso con la gestión y el desarrollo del sector forestal. En los países mediterráneos, donde la capacidad productiva de los bosques naturales es menor y el valor ecológico y social de los mismos predomina en muchas ocasiones, la investigación orientada a la mejora de los métodos de gestión está disminuyendo muy rápidamente. Este es el caso de España, donde este tipo de investigación se ve amenazada por la política científica que ha inducido un giro de los principales grupos de investigación hacia temas más generales que, en ocasiones, tienen que ver con la gestión forestal pero no son capaces de conectar con ella a escala de aplicación. Este hecho, de favorecer a los grupos que trabajan en aspectos generales en lugar de los temas más locales, no es exclusivo de la investigación forestal y se ha visto reforzado por la aplicación de parámetros cuantitativos (número de artículos, factor de impacto, índice h,..) para la evaluación de los científicos en lugar de tener en cuenta aspectos cualitativos de calidad e impacto de los resultados o relativos de la importancia de los parámetros cuantitativos dentro de cada dominio científico concreto. Ante esa falta de conexión entre la investigación y la gestión se han creado durante los últimos años Centros Tecnológicos con la pretensión de transferir conocimientos técnicos a la gestión forestal y paliar la necesidad de la, cada vez más escasa, investigación forestal aplicable a las condiciones locales. Los Centros Tecnológicos están desarrollando un buen papel de estímulo y apoyo a la gestión forestal aplicada a la realidad de cada especie y área geográfica, pero no son suficientes, pues se trata de Centros con poca capacidad para desarrollar investigaciones aptas para generar nuevos 
conocimientos y que en muchos casos se especializan en la provisión de servicios especializados en competencia con empresas de consultoría. Son más bien como Centros "lagos" o Centros recopiladores de conocimientos y "re-elaboradores" de los mismos (cuyo objetivo es la transferencia y la ayuda al sector forestal), pero no es fácil que puedan sustituir a la investigación forestal orientada.

Se está produciendo un desvío progresivo, muy importante cuantitativamente, pero más si tenemos en cuenta su peso relativo sobre los magros recursos disponibles para la investigación, desde la investigación forestal orientada a la gestión, hacia lo que podríamos llamar una investigación forestal centrada en la generación de conocimientos sobre la estructura y dinámica de los sistemas naturales y poco interesada en mejorar el desarrollo económico del sector forestal, cuyos métodos de gestión, en ocasiones, se desconocen y otras veces se desprecian por considerarlos con bajo contenido científico. Por otra parte, la gestión forestal se realiza con bajos presupuestos y requerimientos técnicos, de modo que en muchos casos no se tienen en cuenta avances científicos relevantes. El alejamiento entre investigación y gestión es cada vez mayor, lo que está provocando que en gran medida los grupos de investigación forestal se estén formando al margen de las peculiaridades e intereses de la gestión forestal y con frecuencia muchos de sus miembros tengan problemas para comprender el engranaje entre investigación y gestión. Tal y como apunta Bravo (2015) en este mismo volumen, esta separación entre gestión e investigación forestal, junto con la fragmentación y aislamiento de los grupos de investigación, disminuye nuestra capacidad de investigación, entendida como la capacidad para desarrollar, generar y diseminar ciencia y tecnología, de modo que los esfuerzos realizados no cristalicen en un verdadero avance científico de forma que se facilite su aplicación práctica en la gestión forestal

Esta falta de sincronización puede encontrar sus causas en algunas políticas ajenas al sector forestal. La primera de ellas podría estar relacionada con los costos de instalación, mantenimiento y toma de datos en diseños experimentales, casi a escala real, para poder medir los efectos de determinados tratamientos selvícolas sobre el mayor o menor grado de sostenibilidad del método de gestión al cual asisten, así como su adecuación en dife- rentes condiciones ecológicas, y su viabilidad económica. Estos diseños experimentales consumen mucho trabajo de toma de datos en campo por personal especializado y exigen saber armonizar los resultados cuantitativos derivados del diseño experimental con algunos aspectos sociales locales. Dada la estructura de los sistemas de evaluación científica, en muchos casos es más rentable para un investigador joven especializarse en explotar bases de datos tomadas por otros que en generar y mantener bases de datos a largo plazo. Un cambio radical en la valoración del trabajo científico y en la gestión de bases de datos (derechos de uso, reconocimiento de labores de instalación y mantenimiento,...) ayudaría a fortalecer estas grandes instalaciones de investigación que son las redes de parcelas y ensayos forestales, y que en España están muy bien representadas (ver, entre otros, los trabajos de BrAVO et al., 2004, MONTERO et al., 2004, ROJO et al., 2004). La información obtenida en este tipo de investigación experimental mantiene la generalización que incorpora procedente de los sistemas selvícolas y la dinámica de poblaciones, pero necesita matizaciones importantes que la hagan viable económicamente, o no, según las condiciones locales en que se aplica. El desarrollo de sistemas selvícolas eficientes se basa, en parte, en el conocimiento ecológico. Sin embargo, la aplicación correcta de ese conocimiento depende, muchas veces, del contexto particular. En otras palabras, el puente entre los conocimientos básicos, y el qué, el cuánto y el cómo se incorporan a los métodos selvícolas, se tiene que construir en cada caso particular y aquí instrumentos como la gestión adaptativa (BRAVO, 2009 y 2015) puede ayudar a generar estos puentes, facilitando complicidades entre gestores e investigadores que hagan viable una autentica transferencia de conocimientos.

En las últimas décadas, en España pero también en otras partes del mundo, se han creado grandes bases de datos sobre el medio natural (Inventarios forestales, Inventarios ecológicos, cartografía temática, etc.) que constituyen un soporte inestimable para la investigación forestal. Estos bancos de datos están siendo muy utilizados por los investigadores para generar trabajos de investigación a través de grandes bases de datos numéricos, utilizando diferentes metodologías. Su importancia es grande en el conocimiento de los ecosistemas forestales, pero su aplicación a escala de la gestión 
forestal, a la que antes se hacía referencia, no siempre es posible. Sin embargo, los avances así generados deben ser calibrados para que puedan ser usados en la gestión forestal. Habitualmente, el primer paso (desarrollo científico a partir de grandes bases de datos) por su contenido metodológico, la escala territorial que representan y el carácter general de los resultados, suele ser publicado en revistas de alto impacto generando un efecto positivo sobre la carrera de los investigadores (siguiendo el patrón de evaluación científica cuantitativa que antes hemos comentado). Sin embargo, el segundo paso (calibración de resultados a escala local para poner a disposición de los gestores los avances científi$\cos )$ no encuentra fácil eco en ese tipo de revistas y las que están más accesibles no tienen ese reconocimiento, bien por falta de interés en conseguirlo por parte de sus editores (aunque los trabajos publicados lo merezcan) o bien por la baja calidad del trabajo de revisión de las aportaciones, de forma que en muchos casos lo que se publica carece de un mínimo rigor científico, son resultados parciales o contienen numerosas especulaciones no concordantes con los datos obtenidos.

Urge disponer de herramientas (revistas en español con factor de impacto reconocido internacionalmente como están haciendo nuestros colegas en Iberoamérica, presupuestos orientados, evaluaciones cualitativas de los resultados, sistemas de promoción dentro de la carrera científica que valoren las aportaciones localmente importantes,...) que animen a los investigadores españoles a trabajar en la solución de problemas relevantes localmente. Así mismo, es perentorio que los gestores identifiquen la necesidad de incorporar los avances científicos a su trabajo diario y que enriquezcan la tradición de la gestión forestal con conocimiento nuevo.

\section{EL PAPEL DE LA SOCIEDAD ESPAÑOLA DE CIENCIAS FORESTALES (S.E.C.F.) EN LA INVESTIGACIÓN Y LA DIVULGACIÓN FORESTAL}

La Sociedad Española de Ciencias Forestales (S.E.C.F.) en sus primeros 20 años de andadura, ha realizado una importante labor de equilibrio entre la investigación básica y la aplicada. Naturalmente, no ha podido librarse de la gran tendencia a que antes hacíamos referencia, pero sí ha logrado publicar muchos trabajos de carácter técnico-científico, orientados a la resolución de problemas concretos, que ayudan a la gestión de los bosques españoles. Este tipo de trabajos, con menor difusión internacional que los publicados en revistas internacionales incluidas en el ISI, son muy valorados por los técnicos de los servicios forestales y por las empresas del sector. En este sentido, se puede afirmar que la S.E.C.F. está haciendo una labor importante para acercar la ciencia forestal a la gestión de forma que el conocimiento generado pueda ser transformado en riqueza.

La S.E.C.F. no realiza investigación por sí misma, o lo hace en muy pequeña medida, pero ha encontrado la manera de movilizar a investigadores y técnicos a través de numerosas reuniones organizadas por los más de 20 grupos de trabajo (GT) de la misma, en las que se presentan y discuten temas de interés científico y técnico por investigadores y técnicos que confrontan sus puntos de vista y proponen soluciones viables para ambas partes. Los resultados no siempre logran el nivel científicamente deseable, pero es innegable que están contribuyendo a la divulgación y transferencia de la ciencia forestal más que ninguna otra forma de divulgación y difusión conocidas hasta ahora en el sector forestal.

Somos conscientes de que no todos los conocimientos obtenidos por la investigación son de aplicación inmediata, pero también puede asegurarse que la ciencia forestal cuenta con más conocimientos susceptibles de ser aplicados que aquellos que la técnica cotidiana aplica en la gestión de bosques. Hacen falta investigadores preocupados por orientar sus trabajos a la resolución de problemas reales, de la misma manera que hacen falta técnicos gestores interesados en incorporar los nuevos conocimientos a la gestión, para que la técnica forestal se renueve y el sector se modernice al ritmo que le corresponde. El monte es un valor en alza que necesita cada vez más tecnología, para conseguir una gestión que cada vez es más compleja y que ha de garantizar usos y aprovechamientos compatibles con la conservación, mantenimiento de la diversidad y de la funcionalidad biológica del ecosistema.

La innovación de la gestión forestal que la sociedad exige tiene que nutrirse de la ciencia forestal. Sin investigación no puede haber innova- 
ción y sin innovación de los métodos de gestión no puede haber desarrollo y modernización del sector forestal-medioambiental que debe ser, cada vez más, un sector tecnológicamente avanzado.

En términos cuantitativos, la S.E.C.F. ha publicado 39 volúmenes de los conocidos "Cuadernos de la Sociedad Española de Ciencias Forestales", incluyendo este último número que aparece ahora, y que corresponde a las conferencias y ponencias invitadas del $6^{\circ}$ Congreso Forestal Español. Este será el último volumen que se publica impreso en papel, el numero 40 aparece ya en formato digital. Estamos seguros que esta nueva etapa de los Cuadernos de la Sociedad vera reforzados los niveles de consulta y de lectura de sus contenidos. En los primeros 39 números se han publicado 1053 trabajos repartidos entre los temas a que se refiere los grupos de trabajo de la S.E.C.F. (Tabla 1).

\begin{tabular}{|c|c|c|c|}
\hline Grupo de Trabajo & Año de edición & $\mathrm{N}^{\circ}$ de cuaderno & $\mathrm{N}^{\circ}$ de artículos \\
\hline Ordenación & $\begin{array}{l}1995 \\
1998 \\
2001 \\
2008 \\
2013\end{array}$ & $\begin{array}{c}1 \\
6 \\
11 \\
27 \\
37\end{array}$ & $\begin{array}{l}37 \\
20 \\
22 \\
20 \\
12\end{array}$ \\
\hline Cambio climático y Fitoclimatología & $\begin{array}{l}1995 \\
1998 \\
2001\end{array}$ & $\begin{array}{c}2 \\
7 \\
12\end{array}$ & $\begin{array}{c}7 \\
9 \\
14\end{array}$ \\
\hline Selvicultura & $\begin{array}{l}1996 \\
2000 \\
2003 \\
2007\end{array}$ & $\begin{array}{c}3 \\
10 \\
15 \text { y } 15 b \\
21\end{array}$ & $\begin{array}{c}17 \\
27 \\
27-4 \\
24\end{array}$ \\
\hline Repoblaciones & $\begin{array}{l}1997 \\
2004 \\
2008\end{array}$ & $\begin{array}{c}4 \\
17 \\
28\end{array}$ & $\begin{array}{l}14 \\
38 \\
47\end{array}$ \\
\hline Genética forestal & $\begin{array}{l}1997 \\
2008\end{array}$ & $\begin{array}{c}5 \\
24\end{array}$ & $\begin{array}{l}34 \\
21\end{array}$ \\
\hline Historia forestal & $\begin{array}{l}1999 \\
2003 \\
2009 \\
2013\end{array}$ & $\begin{array}{c}8 \\
16 \\
30 \\
38\end{array}$ & $\begin{array}{l}11 \\
49 \\
51 \\
24\end{array}$ \\
\hline Fuegos Forestales & 2000 & 9 & 28 \\
\hline Hidrología & $\begin{array}{l}2002 \\
2011\end{array}$ & $\begin{array}{l}13 \\
32\end{array}$ & $\begin{array}{l}24 \\
21\end{array}$ \\
\hline Sistemas Agroforestales & $\begin{array}{l}2002 \\
2007 \\
2011\end{array}$ & $\begin{array}{l}14 \\
22 \\
33\end{array}$ & $\begin{array}{l}19 \\
26 \\
22\end{array}$ \\
\hline Modelización forestal & $\begin{array}{l}2004 \\
2007 \\
2012\end{array}$ & $\begin{array}{l}18 \\
23 \\
34\end{array}$ & $\begin{array}{l}48 \\
34 \\
38\end{array}$ \\
\hline Inventario y teledetección & 2005 & 19 & 32 \\
\hline Ecología forestal & $\begin{array}{l}2005 \\
2008 \\
2012\end{array}$ & $\begin{array}{c}20 \text { y } 20 b \\
25 \\
35\end{array}$ & $\begin{array}{c}36-4 \\
63 \\
27\end{array}$ \\
\hline Sanidad forestal & $\begin{array}{l}2008 \\
2012\end{array}$ & $\begin{array}{l}26 \\
36\end{array}$ & $\begin{array}{l}29 \\
26\end{array}$ \\
\hline SIG-Teledetección & 2009 & 29 & 10 \\
\hline Conferencias y ponencias del $5^{\circ} \mathrm{CFE}$ & 2010 & 31 & 14 \\
\hline Conferencias y ponencias del $6^{\circ} \mathrm{CFE}$ & $2015^{*}$ & 39 & 23 \\
\hline TOTAL & 1995-2015 & 39 & 1053 \\
\hline
\end{tabular}

Tabla 2. Número de trabajos publicados en los Cuadernos de la Sociedad Española de Ciencias Forestales (1993-2015) 


\begin{tabular}{|l|c|c|c|}
\hline Congreso & Año celebración & Documento & $\mathbf{N}^{\mathbf{o}}$ Trabajos \\
\hline $1^{\text {er }} \mathrm{CFE}$ & 1993 & Actas & 358 \\
\hline $2^{\text {o }} \mathrm{CFE}$ & 1997 & Actas & 512 \\
\hline $3^{\text {er }} \mathrm{CFE}$ & 2001 & Actas & 615 \\
\hline $4^{\circ} \mathrm{CFE}$ & 2005 & Actas & 575 \\
\hline $5^{\circ} \mathrm{CFE}$ & 2009 & Actas & 687 \\
\hline $6^{\circ} \mathrm{CFE}$ & 2013 & Actas & 636 \\
\hline TOTAL & $\mathbf{1 9 9 3 - 2 0 1 3}$ & & $\mathbf{3 3 8 3}$ \\
\hline
\end{tabular}

Tabla 2. Número de trabajos publicados en las actas de los congresos forestales (1993-2013)

La otra gran aportación bibliográfica de la S.E.C.F., junto con el Informe de Situación de los Bosques y el Sector Forestal en España, procede de los trabajos presentados y publicados en las actas del Congreso Forestal Español, que se celebra cada 4 años. En los seis congresos celebrados hasta la fecha se han publicado 3383 trabajos de contenido técnico-científico (Tabla 2), más o menos relacionados con la gestión forestal y con las nuevas grandes líneas de investigación de los últimos 20 años, que, grosso modo, coinciden con los "lemas" de cada congreso, salvo el primero que, en 1993, que no llevó asociado un lema especial. Los restantes, en 1997, "Montes del futuro: Respuesta ante un mundo en cambio", en 2001, "Montes para la Sociedad del nuevo milenio", en 2005, “La Ciencia Forestal: Respuestas para la Sostenibilidad", en 2009, “Montes y Sociedad: Saber qué hacer" y por último en 2013,"Montes, Servicios y Desarrollo Rural". Estos lemas ponen de manifiesto el interés de la S.E.C.F. por desarrollar una ciencia orientada a las necesidades y exigencias de la sociedad española. Otras publicaciones como el Diccionario Forestal publicado en 2005 y la reedición de monografías de interés técnico o histórico completan la obra editorial de la S.E.C.F.

En fin, la publicación de más de 4400 trabajos sobre temas forestales en los últimos 20 años, supones un récor que, incluso en términos numéricos, debe de igualar o superar a todo lo publicado anteriormente en nuestro país sobre aspectos forestales-medioambientales. No tenemos datos sobre el nivel de consulta y lectura del banco bibliográfico de la S.E.C.F., pero es evidente el esfuerzo que ha hecho esta Sociedad para poner la ciencia y la técnica forestales en manos de las profesiones interesados.

Con ser mucho lo hecho, los que componemos la S.E.C.F. no podemos darnos por satisfechos. Aún debemos, y podemos, hacer más. Entre lo que más urge es facilitar no solo la interacción entre investigadores y gestores, sino la correcta valoración de los trabajos más aplicados, que con el más alto rigor puedan ser publicados a través de los instrumentos de que dispongamos en cada momento incluyendo, si es que así lo solicitan los socios de la S.E.C.F., revistas científicas de relevancia e impacto, bancos de datos abiertos y enlazados, informes de situación y programas de prospectiva científica para orientar, sobre todo, a los grupos e investigadores emergentes.

\section{Bibliografía}

Bravo, F.; Ordóñez， C.; Lizarralde, I.; Bravo-Oviedo, A.; Guerra, B.; Peso, C. DEL; DOMÍngueZ, M. Y Osorio, L.F.; 2004. Red de parcelas y experimentos del grupo de investigación sobre gestión forestal sostenible de la ETS de Ingenierías Agrarias de Palencia (Universidad de Valladolid). Cuad. Soc. Esp. Cienc. For. 18: 237-242.

BRAVO, F.; 2009. Adaptive Forest Management: Learning by doing in forestry. In: M. Palahí, Y. Birot, F. Bravo \& E. Górriz (eds.), Modelling, Valuing and Managing Mediterranean Forest Ecosystems for NonTimber Goods and Services. EFI Proceedings 57: 111-118. Joenssuu.

Bravo, F.; 2015. ¿Se puede generar conocimiento mediante la gestión adaptativa para 
fundamentar un cambio de paradigma de la selvicultura?. Cuad. Soc. Esp. Cienc. For. 39: 131-138.

EUROPEAN COMMISSION; 2010, 2012, 2015. Citados por Campos, P. (2011). Valor económico y ambiental de los bosques. Seminario, Situación actual de los bosques: retos y oportunidades. UIMP, Santander, 1-5 de agosto de 2011.

MARTínez DE ARANO, I.; 2011. Visión de la realidad del uso de la biomasa en Europa: http://www.usse-eu.org/Biomasa/vision-delarealidad-del-uso-de-la-biomasa-con-finesenergeticos-eneuropa.

Montero, G.; Madrigal, G.; Ruiz-Peinado, R. Y BACHILLER, A.; 2004. Red de parcelas experimentales permanentes del CIFORINIA. Cuad. Soc. Esp. Cienc. For. 18: 229236.

Montero, G.; Rojo, A. y HernándezFERNÁNDEZ DE RoJAS, A.; 1993. Teoría y práctica de la Selvicultura. En: F.J. SilvaPando \& G. Vega Alonso (eds.), Congreso Forestal Español-Lourizán 1993, II: 438444.Grapol. Vigo.

Rojo, A.; Rodríguez SoAlleiro, R.; SÁnchez Rodríguez, F.; CASTEdo, F. Y GRANDAS, J.A.; 2004. Dispositivos experimentales permanentes de la unidad de gestión forestal sostenible para el estudio de modelos de crecimiento forestal en Galicia. Cuad. Soc. Esp. Cienc. For. 18: 255-260. 